\title{
STABILOGRAPHY A RELIABLE METHOD FOR MEASUREMENT OF POSTURAL BALANCE IN PATIENT AFTER ANTERIOR CRUCIATE LIGAMENT RECONSTRUCTION
}

\author{
Tanya Grueva, Katherina Stambolieva
}

Summary

The purpose of the present report was to observe the dynamics of postural balance during unilateral stance with the help of stabilography method in patients after anterior cruciate ligament reconstruction during their 6 months postoperative recovery period.

Methodology. Forty patients divided in 2 groups, experimental and control ones, have been investigated. The two groups were formed according to the starting day with physiotherapy and the type of exercises in the treatment. Computerized stabilography system ("CAT 2004" Plovdiv/Bulgaria) was used to detect the results at the $1^{\text {st }}, 3^{\text {rd }}$ and $6^{\text {th }}$ month of the physiotherapy treatment. The measurement included quiet unilateral stance with eyes open and eyes closed. The tests were done both on the stable platform, as well as on a foam block with the operated and non-operated leg.

Results. The patients from the experimental group have shown better balance in unilateral stance on the operated leg on the stable platform as well as on the unstable one already at the $3^{\text {rd }}$ month postoperatively. That significant difference was maintained till the $6^{\text {th }}$ postoperative month. Apart of that the patients from the same group have demonstrated better performance with the non-operated leg, which shows better general conditioning with the lower limbs at all. Conclusion. The results show that the stabilography method can be considered as a reliable one for measuring postural balance discrepancies in patients with lower extremity injuries.

Key words: stabilography, postural balance

Introduction. Computer based stabilography system is made to investigate the postural balance changes while maintaining upright position (Hoffman et al., 1999). It can be used to determine different postural strategies in healthy subjects as well as for patients with different pathologies of the musculo skeletal and vestibular systems (Ben Moussa et al., 2009). As we incorporated specific proprioceptive exercises, responsible for the postural balance, in early postoperative treatment in patients after anterior cruciate ligament (ACL) reconstruction (Mohamaddi et al., 2012), we decided to implement the measurement of the static postural balance with the stabilography platform and to check the reliability and validity of the test in patients with that pathology.

The purpose of the present report was to observe the dynamics of postural balance during unilateral stance with the help of stabilography method in patients after ACL reconstruction during their 6 months postoperative recovery period.

Methodology. Forty patients divided in 2 groups, experimental (EG) and control (CG) ones, have been investigated. The two groups were formed according to the starting day with physiotherapy and the type of exercises in the treatment. The phys- iotherapy treatment for the patients from the EG included proprioceptive and close kinetic chain exercises, which were performed still in the early postoperative days (Harrison et al., 1994). While for the patients from the CG these specific exercises were done when they were allowed to do some weight bearing of the operated leg, approximately 30 days after the reconstruction. Computerized stabilography system ("CAT 2004" Plovdiv/Bulgaria) was used to measure the changes in the parameters of the postural balance of the patients while they were maintaining unilateral stance. The system includes stable platform and adapted software (Stambolieva, 2007). The displacements of foot pressure center in both medio-lateral (ML) and anterior-posterior (AP) directions were registered. The following parameters were detected: mean amplitude (MA) and mean velocity $(\mathrm{MV})$. It is presented in the so called stabilograms, fig. 1. Two analogue signals were digitized with a sampling interval of $10 \mathrm{~ms}$ and filtered with a digital Hamming low-pass filter with cut-off frequency of $10 \mathrm{~Hz}$ in order to remove the high frequency noise and sampling error.

The dynamics of postural sways for both groups were statistically processed by monofactor dispersion analysis (ANOVA), where the factor is Time 


\section{POSTUROGRAPHIC PARAMETERS}

\section{Stabilograms}

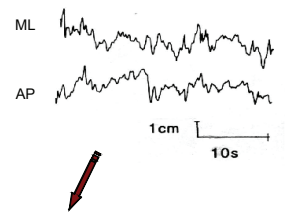

Mean amplitude $[\mathrm{cm}]-\mathrm{MA}_{\mathrm{ML}}$ and $\mathrm{MA}_{\mathrm{AP}}$ Mean velocity $[\mathrm{cm} / \mathrm{s}]-\mathrm{MV}_{\mathrm{ML}}$ and $\mathrm{MV}_{\mathrm{AP}}$

\section{Figure 1}

(T). While the internal group discrepancies were statistically processed by nonparametric Wilcoxon test. The measurement itself was done directly on the platform (stable surface) (figure 2), as well as on a foam block placed on the platform (unstable surface) (figure 3), both conditions performed with the operated and non-operated leg.

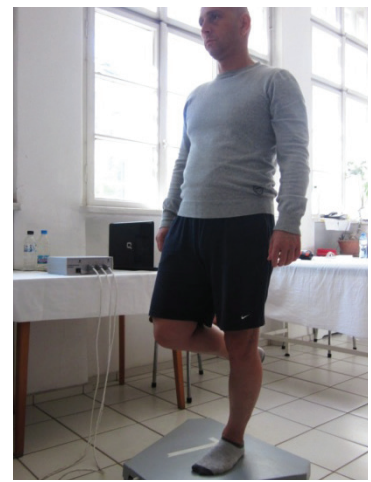

Figure 2

Stable surface

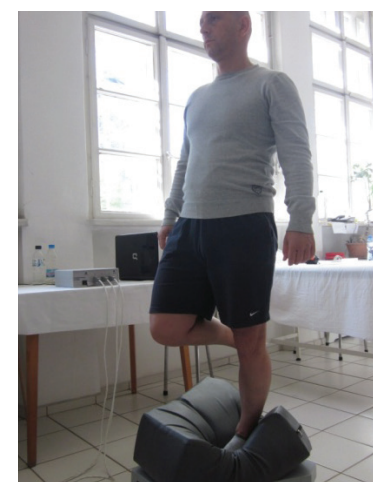

Figure 3

Unstable surface
All tests were done with eyes open (EO) and with eyes closed (EC). In that way, the measurement was done at $1^{\text {st }}, 3^{\text {rd }}$ and $6^{\text {th }}$ postoperative month for the patients from the EG, while for the ones from the CG were done only at $3^{\text {rd }}$ and $6^{\text {th }}$ month. Some of the patients from the CG still could not put weight and stand on the operated leg, for others, performing these tests was too complicated task.

\section{Results.}

Unilateral stance on a stable surface.

There was not much differences between the groups in the oscillation of the mean amplitude of postural sway while standing on a stable surface with eyes open both for operated and non-operated leg. The results for both groups were close, only at the $3^{\text {rd }}$ postoperative month there was a significant difference in AP direction, with lower oscillations for the EG (figure 4).

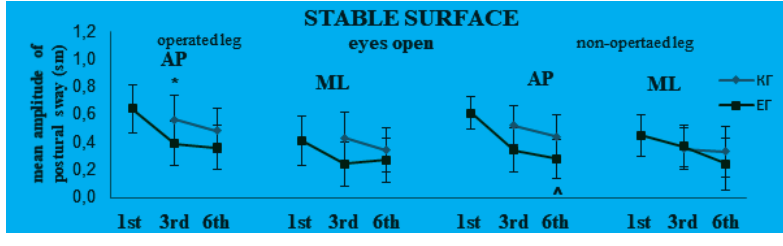

Figure 4

${ }^{*}$ St. Significance between groups $(p<0,05) ; \wedge$ St. Significance between $1^{\text {st }}$ and other measurements within group $(\mathrm{p}<0,05)$

When the vision was eliminated the mean amplitude postural sways for the non-operated leg increased for both groups, but in lesser degree for the patients from the EG. In the time, it was observed a systematical decrease of the oscillation for both groups. While standing on the operated leg, the patients from the EG showed significant lesser oscillation of the mean amplitude, which was statistically confirmed at the $3^{\text {rd }}$ and $6^{\text {th }}$ month in both orthogonal axes. The starting values in AP and ML direction for the $\mathrm{EG}$ at the $1^{\text {st }}$ monitoring month were even lower than the data collected for the CG at the $3^{\text {rd }}$ monitoring month (figure 5).

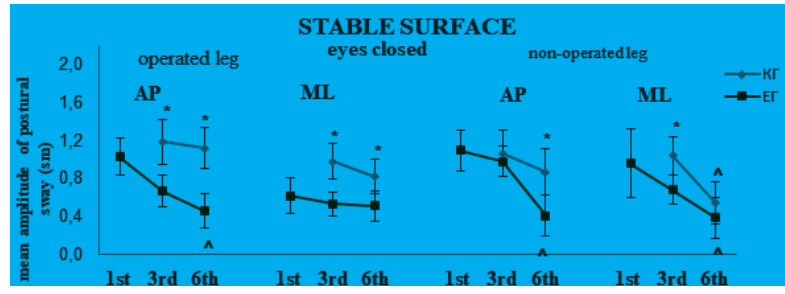

Figure 5

${ }^{*}$ St. Significance between groups $(\mathrm{p}<0,05) ; \wedge$ St. Significance between $1^{\text {st }}$ and other measurements within group $(\mathrm{p}<0,05)$

The mean velocity of postural sway while standing either on the non-operated or operated leg, on a stable surface, with eyes open, was very close between the groups throughout the monitoring periods, and that for both directions (Figure 6)

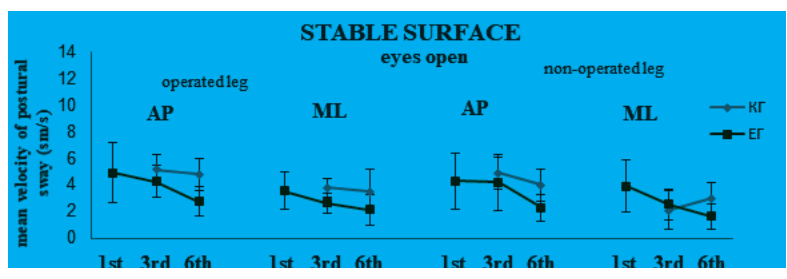

Figure 6

When reducing the visual input, the results was considerably different. The velocity sway in both 
orthogonal axes, of the non-operated leg, for the EG was lower for the whole period, but it was statistically significant only at the $6^{\text {th }}$ postoperative month. Also, it was significantly established a systematical drop of the values in the EG in every second control period. Furthermore, velocity sways in unilateral stance on the operated leg had a different point. For the EG the values of the $1^{\text {st }}$ and $3^{\text {rd }}$ month were very close, they lowered significantly still at the $6^{\text {th }}$ monitoring month, where there was also statistical difference between the groups, for AP plane. For the CG, the velocity sway was lower in the final control period, but it was statistically supported only in ML axes (figure 7).

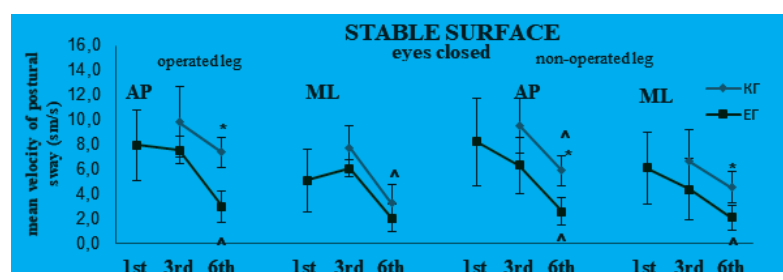

Figure 7

${ }^{\star}$ St. Significance between groups $(p<0,05) ; \wedge$ St. Significance between $1^{\text {st }}$ and other measurements within group $(\mathrm{p}<0,05)$

It was observed that the parameters of postural sway in unilateral stance on the operated leg, on a stable surface when eyes were open, did not differ much between the groups through the measured period. Even though they lower in every next control period, the changes are very little. With reducing the vision, patients from both groups showed different strategies to maintain quiet unilateral postural balance. The posturographic parameters increase for both groups, but that was observed significantly more for the CG in both orthogonal planes. The patients from the EG showed more stable unilateral stance on the operated leg still on the $3^{\text {rd }}$ control month and that maintained till the $6^{\text {th }}$ one, especially in AP plane.

\section{Unilateral stance on an unstable surface.}

The mean amplitude of postural sway in standing on the operated leg on a foam block, when eyes were open was much more expressed considering the value in standing on the non-operated leg, and that for both groups. A significant difference was seen between the groups in the time, while maintaining balance on the operated leg. The dynamics of oscillations is minimal and the values between the groups in non-operated leg were very close (fig- ure 8). The condition of closed eyes did not affect the tendency between the groups while standing on the operated leg. But it has changed while standing on the non-operated one. The amplitude oscillations have

increased for both groups, as for the patients from the EG the values were significantly lower in ML plane at the $3^{\text {rd }}$ monitoring month and in AP plane at the $6^{\text {th }}$ month, comparing with the CG. Also, the final results for the EG, compared to the starting ones were much lower, statistically supported, in both orthogonal axes (figure 9).

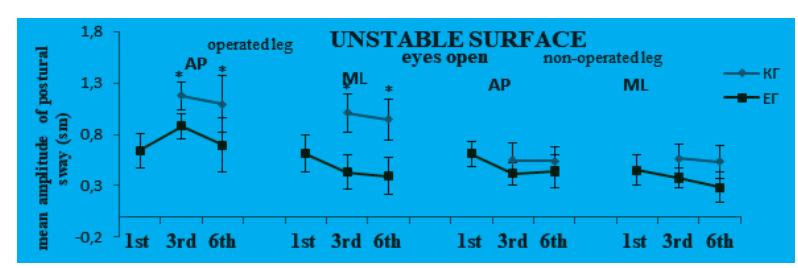

Figure 8

${ }^{\star}$ St. Significance between groups $(\mathrm{p}<0,05)$

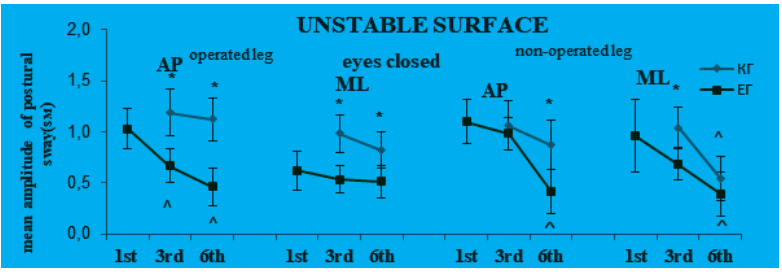

Figure 9

${ }^{*}$ St. Significance between groups $(p<0,05)$; $\wedge$ St. Significance between $1^{\text {st }}$ and other measurements within group $(\mathrm{p}<0,05)$

Under the same experimental circumstances, the mean velocity sway was comparatively equal for the operated and non-operated leg and that for both groups. Statistical significant differences between the groups were observed mainly at the $6^{\text {th }}$ control month. The lower values were for the EG. Within the groups there was significant differences between the starting and final values in both planes for both legs, for the patients from the EG. For the CG, it was in ML plane for the operated leg and in AP plane for the non-operated leg (figure 10).

Like in the other experimental conditions here with closing eyes standing on one leg on a foam block the velocity postural sway increased for both groups, but with significant lower values for the patients in the EG. Standing either on the operated or on the non-operated leg statistical significance could be seen already at the $3^{\text {rd }}$ control month in AP plane 
and in both orthogonal axes at the $6^{\text {th }}$ monitoring month. Both groups showed significant progressive decrease of velocity sway values in the time, especially in AP plane, and for the EG this is observed still at $3^{\text {rd }}$ postoperative month (figure 11).

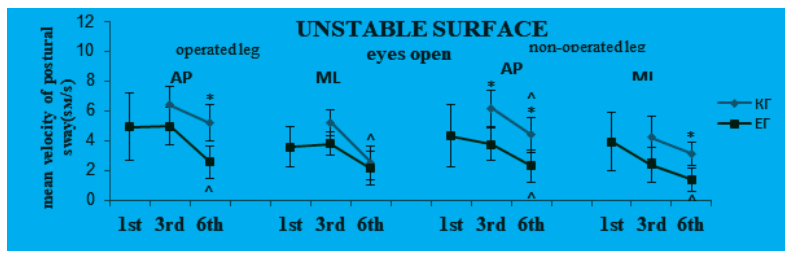

Figure 10

${ }^{*}$ St. Significance between groups $(\mathrm{p}<0,05) ; \wedge$ St. Significance between $1^{\text {st }}$ and other measurements within group $(\mathrm{p}<0,05)$

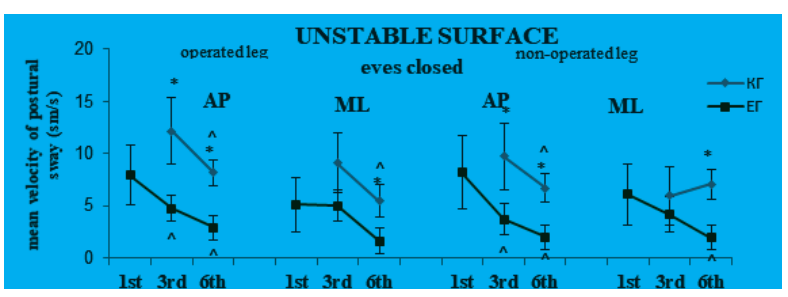

Figure 11

${ }^{\star}$ St. Significance between groups $(\mathrm{p}<0,05) ; \wedge$ St. Significance between $1^{\text {st }}$ and other measurements within group $(\mathrm{p}<0,05)$

Overall the patients from the EG were dealing better with the given task of maintaining unilateral stance on unstable surface, meaning on a foam block. The patients from the CG showed significantly higher amplitude and velocity postural sway values during the measured period. Even though they showed positive dynamics of postural sway during the time, those discrepancies between the groups were well observed at the $6^{\text {th }}$ control month in both orthogonal axes, as well as with eyes open, as with eyes closed.

In general, the patients from the EG have shown better balance in unilateral stance on the operated leg on the stable platform as well as on the unstable one already at the $3^{\text {rd }}$ month postoperatively. That significant difference was maintained till the $6^{\text {th }}$ postoperative month. Apart of that the patients from the same group have demonstrated better performance with the non-operated leg, which shows better general conditioning with the lower limbs at all.

Conclusion. The results show that the stabilography method can be used effectively for scientific investigations, as well as for clinical practice, to determine and follow the dynamics of postural balance in patients with ACL disorders. Also, it can be considered as a reliable tool for measuring postural balance discrepancies in patients with lower extremity injuries.

\section{References:}

Ben Moussa AZ, Zouita S, Dziri C et al. Single-limb assessment of postural stability and knee functional outcome two years after ACL reconstruction. Ann Phys Rehabil Med (2009) 52:475-484

Harrison EL, Duenken N, Dunlop R et al. Evaluation of single-limb standing following ACL surgery and rehabilitation. Phys Ther (1994) 74:245-252

Hoffman M, Scharder J, Koceja D. An investigation of postural control in postoperative ACL reconstruction patients. J Athl train (1999) 34:130-136

Mohammadi F, Salavati M, Akhbari B, Mazaheri M, Khorrami M, Negahban H. Static and dynamic postural control in competitive athletes after ACL reconstruction and controls. Knee Surg Sports Traumatol Arthrosc (2012) 20:1603-1610

Stambolieva K. Computerized stabilographic measurement of the functional status to the vestibular analizator $\mathrm{PhD}$ disertation/ Sofia 2007.

\section{Corresponding author:}

Chef assistant, Tanya Grueva-Pancheva, $\mathrm{PhD}$

tgrueva2000@gmail.com

Sofia 1000/str.Gurguliat 1

NSA "Vassil Levski"

Kinesitherapy faculty

TM of the kinesitherapy 